\title{
Nutritional Characterization of Medicago sativa (Alfalfa) for Feeding to Ruminants
}

\section{Caracterización Nutricional de henos de Medicago sativa (Alfalfa) para la alimentación de Rumiantes}

\author{
López FA, Vargas PT, Yánez SJ, and Haro MC
}

Carrera de Zootecnia, Facultad de Ciencias Pecuarias, Escuela Superior de Chimborazo, Riobamba, Ecuador

I International Seminar of Livestock and Agroindustrial Production ESPOCH 2020

Corresponding Author:

P. Toalombo Vargas

ptoalombo@espoch.edu.ec

Published: 2 September 2021

Production and Hosting by

Knowledge $E$

(c) F. Almeida López et al. This article is distributed under the terms of the Creative Commons Attribution License, which permits unrestricted use and redistribution provided that the original author and source are credited.

\section{A OPEN ACCESS}

\section{Abstract}

This study aimed to analyze the nutritional characteristics of Medicago sativa (Alfalfa) hay for the feeding of ruminants in the central zone of the Sierra Region. The study was conducted in the Laboratory of the Faculty of Pecaurias Sciences of the Higher Polytechnic School of Chimborazo. Cutting was carried out for 60 days in different areas of the central Sierra region. Data were analyzed using descriptive statistics. Results of the bromatological analysis showed that the average total humidity was $11.12 \pm 0.20 \%$ in (Rp-00542) while the minimum was $7.79 \pm$ $0.05 \%$ in (Rp-00224). Values for dry matter ranged between $91.25 \pm 0.03 \%(\mathrm{Rp} 336)$ and 88.53 $\pm 0.09 \%$ (Rp492); dry base ash ranged between $12.51 \pm 0.08 \%(\mathrm{Rp} 500)$ and $9.16 \pm 0.08 \%$ (Rp521); dry organic matter ranged between $90.84 \pm 0.13 \%(R p 521)$ and $87.49 \pm 0.08 \%(R p 521)$; and dry crude protein ranged between $22.44 \pm 0.26 \%$ (Rp492) and $18.73 \pm 0.08 \%$ (Rp336). The following values were also obtained: $1.94 \pm 0.93 \%$ dry ethereal extract; $32.06 \pm 2.11 \%$ dry nitrogen free extract; $46.96 \pm 3.78 \%$ dry neutral detergent fiber; $40.67 \pm 3.36 \%$ dry acid detergent fiber; and $8.23 \pm 0.64 \%$ dry lignin detergent acid. Therefore, it can be determined that forage mixture provides all of the nutrients to the species of zootechnical interest.

Keywords: Medicago sativa, alfalfa, sierra centro, rumiants.

\section{Resumen}

En el Laboratorio de la Facultad de Ciencias Pecaurias de la Escuela Superior Politécnica de Chimborazo, se realizó la Caracterización Nutricional de henos de Medicago sativa (Alfalfa) para la alimentación de Rumiantes en la zona centro de la Región Sierra; Mediante estadística descriptiva se obtuvo los siguientes resultados del análisis bromatológico a los 60 días de corte en las diferentes zonas de la región Sierra - Centro, se puede observar que el mayor promedio de la humedad total fue $11,12 \pm 0,20 \%$ en (Rp-00542) mientras que el menos fue 7,79 \pm 0,05\% en (Rp-00224); para Materia Seca-Total los valores se obtuvieron entre 91,25 \pm 0,03\% (Rp336) y 88,53 \pm 0,09\% (Rp492); Cenizas base seca 12,51 $\pm 0,08 \%(R p 500)$ y $9,16 \pm$ 0,08\% (Rp521); el mayor valor para \% Materia Orgánica Base seca fue de 90,84 \pm 0,13\% (Rp521) y el menor con 87,49 \pm 0,08\% (Rp521); para \% Proteína cruda base seca la mejor respuesta fue $22,44 \pm 0,26$ (Rp492) \% y el menor 18,73 \pm 0,08 (Rp336), Fibra cruda base seca; 1,94 \pm 0,93\% Extracto etéreo base seca; 32,06 \pm 2,11\% Extracto Libre de Nitrógeno base seca; 46,96 $\pm 3,78 \%$ Fibra Detergente Neutro base seca; 40,67 $\pm 3,36 \%$ Fibra Detergente Acida base seca; $8,23 \pm 0,64 \%$ Lignina Detergente Acida base seca. Por lo que se puede determinar que la mezcla forrajera aporta todos los nutrientes a todas las especies de interés zootécnico. 


\section{Introducción}

La capacidad y calidad de producción de las especies forrajeras es resultado de su adaptación a condiciones climáticas de cada ecoregión, del manejo y fertilización (ALVIM). Por lo que se debe considerar la reducida calidad nutricional de las pasturas, debido principalmente al manejo deficiente de las praderas, relacionado con los tiempos de uso y descanso, renovación de praderas, y a su vez, al establecimiento de pastizales con especies mejoradas [1] Cada ganadería presenta su diferente sistema, por lo que la condición climática para la producción de leche se encuentra asociada a regiones templadas.

El ganado vacuno es muy variable respecto a la alimentación, pero al ser rumiante, el pasto es la base fundamental de su alimentación [2] pero no logra cubrir los requerimientos nutricionales para su mantenimiento y productividad, por lo que es importante conocer nuevas alternativas de alimentación y sus niveles de utilización [3]. Los componentes claves en la productividad de un sistema de pastoreo son la materia seca (MS) producida por unidad de área, su contenido de energía y proteína digestible y su patrón de crecimiento [4].

Las leguminosas, aumentan el consumo voluntario y mejoran el funcionamiento del rumen [5], la alfalfa (Medicago sativa) es el principal forraje para la producción de leche a nivel mundial, dentro de las forrajeras cultivadas, las leguminosas del género Medicago son las más utilizadas [6]. La alfalfa, por su alto rendimiento y contenido de proteína, vitaminas, minerales (especialmente calcio) y bajo porcentaje de fibra, es excelente para la producción de leche; además ayuda a enriquecer el suelo por su capacidad de fijar nitrógeno atmosférico en asociación [7].

El rendimiento y calidad nutricional del forraje define, en gran parte, la respuesta animal, el suelo debe presentar una adecuada incorporación de materia orgánica y de nutrientes que permitan un proceso de producción eficiente. De manera tradicional se ha empleado la fertilización química que aporta nitrógeno, fósforo y potasio, la cual, tiene un efecto a corto plazo [8] y puede afectar las características fisicoquímicas y microbiológicas de suelo; debido a que el uso excesivo de este elemento incrementa los procesos degradativos por la disminución de la fracción orgánica y desequilibra el ciclo global del nitrógeno y del fósforo $[9,10]$. Por lo que el objetivo de la presente investigación fue realizar la caracterización nutricional de henos de Medicago sativa (Alfalfa) para la alimentación de rumiantes.

\section{Materiales y Métodos}

Se utilizaron muestras de heno de alfalfa de nueve sitios pertenecientes a las provincias de Chimborazo y Tungurahua. Los Sitios escogidos para el estudio, fueron. San Juan, San Gerardo, San Andrés, Licto y Calpi (Provincia de Chimborazo). Cuchibamba, Totoras, Huachi Chico y Quizapincha (Provincia de Tungurahua).

El estudio se realizó en la sierra centro del Ecuador, con un clima Temperado húmedo. Este se extiende por los flancos internos y externos de la cordillera de los Andes. Abarca las tierras comprendidas entre los 2500 y los 3200 m de altura. La mayoría de 
las capitales de provincias de la Sierra se ubica en él. Su pluviosidad es mayor que en los anteriores pisos climáticos y llega hasta los $2000 \mathrm{~mm}$ anuales. La temperatura promedio se encuentra entre 12 y $20^{\circ} \mathrm{C}$. (The greatest WordPress.com site in all the land. La Sierra Ecuatoriana. 2.020.)
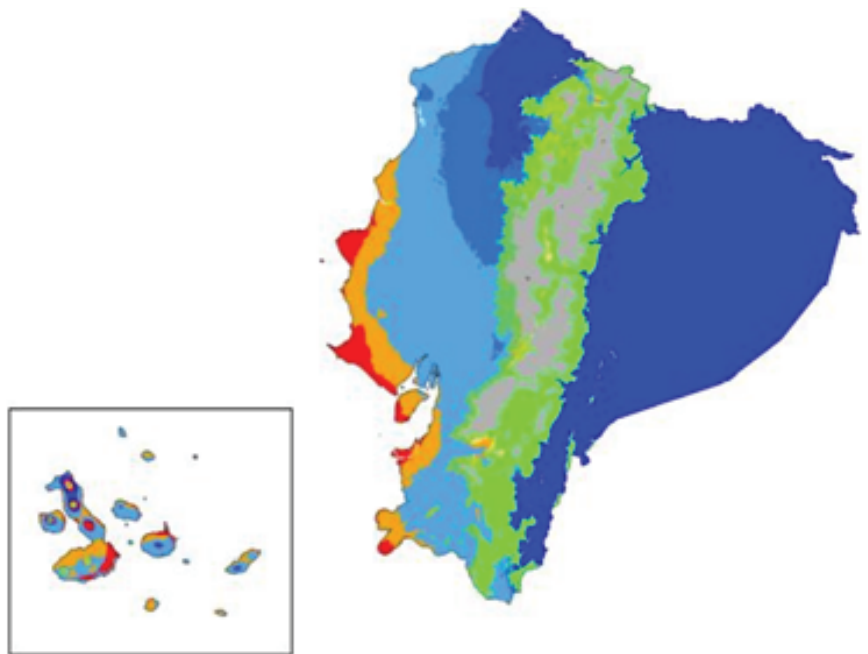

Tropical, rainforest (Af) Tropical, monsoon (Am) Tropical, savannah (Aw) Arid, desert, hot (BWh) Arid, desert, cold (BWk) Arid, steppe, hot (BSh) Arid, steppe, cold (BSk)

Temperate, dry summer, warm summer (Csb) Temperate, dry summer, cold summer (CSC) Temperate, dry winter, warm summer (Cwb) Temperate, dry winter, cold summer (Cwc) Temperate, no dry season, hot summer (Cta) Temperate, no dry season, warm summer (Cfo) Temperate, no dry season, cold summer (Cfc) Polar, tundra (ET) Polar, frost (EF)

Figure 1

Beck, H. E.; Zimmermann, N.E.; McVicar, T.R.; Vergopolan, N.; Berg, A.; Wood, E.F. Present and future Köppen-Geiger climate classification maps at 1-km resolution. Sci. Data 2018, 5, 180214.

Los análisis de laboratorio se realizaron en el Laboratorio de Nutrición Animal de la Facultad de Ciencias Pecuarias de la ESPOCH, Riobamba. Las diferentes pruebas se ejecutaron mediante la aplicación de 2 métodos analíticos internacionales oficiales.

\subsection{Official methods of analysis $-13^{\text {th }}$ edition, 1984}

Los métodos oficiales se designan como acción primera y final, en algunos casos, de procedimientos. Un primer método de acción ha sido objeto de un estudio colaborativo, ha sido recomendado por el comité de arbitraje y métodos general apropiado. ha sido aprobada la primera acción provisional por el presidente de la junta de métodos oficiales, y ha sido adoptada oficialmente por los miembros de la asociación en una reunión anual $[11,12]$.

\subsection{Food and nutrition paper $14 / 7$ Roma, 1986}

Los métodos y procedimientos analíticos aquí descritos. Están diseñados por medio de manuales que están diseñados para ser realizados por personal en un laboratorio debidamente equipado. En común con muchos procedimientos de laboratorio, los métodos citados con frecuencia involucran materiales peligrosos. Para la correcta y segura ejecución de estos métodos es esencial que el personal de laboratorio siga las normas de seguridad procedimientos para el manejo de materiales peligrosos. Si bien se ha ejercido el mayor cuidado en la preparación de esta información, Los métodos tampoco deben considerarse oficiales porque de su inclusión en este Manual. Son 
simplemente métodos que se han encontrado por experiencia para ser utilizables en el laboratorio medio (FAO.1986).

\subsection{Determinación de humedad inicial}

La determinación de humedad inicial se realiza siguiendo los procesos de las técnicas antes mencionadas. Al determinar y cuantificar la humedad inicial presente en una muestra de alimento con un alto contenido de humedad como pastos y forrajes, ensilajes, henolajes arbustivas y raíces mediante la metodología del análisis proximal para evaluar y asegurar la calidad del forraje.

Este método se basa en la volatilización del agua a causa del calor, hasta que se haya eliminado alrededor de un $90 \%$ de agua aplicando para ello una temperatura de 65 grados centígrados.

\subsubsection{Cálculo y expresión de resultados}

$\%$ Materia seca $=\frac{(\text { Peso de la funda }+ \text { Muestra Seca })-(\text { Peso de la funda sola })}{(\text { Peso de la funda }+ \text { Muestra fresca })-(\text { Peso de la funda sola })} \times 100$ \%Humedad inicial $=100-\%$ Materia seca.

\subsection{Determinación de extracto etéreo}

Se calculó la determinación de extracto etéreo mediante el uso de las técnicas descritas anteriormente, esto nos permite determinar y cuantificar la cantidad de extracto etéreo presente en una muestra de alimento seco o de un forraje, mediante la metodología del análisis proximal para evaluar la calidad del forraje o el alimento completo. El hexano se evapora y se condensa continuamente y al pasar a través de la muestra extrae materiales solubles en el solvente orgánico. El extracto se recoge en un beaker y cuando el proceso se completa el hexano se destila y se recolecta en otro recipiente, y la grasa que queda en el beaker se seca y se pesa.

\subsubsection{Cálculo y expresión de resultados}

$$
\begin{gathered}
\% \text { E.E. }=\frac{(\text { Peso beaker }+ \text { E.E. })-(\text { Peso beaker solo })}{(\text { Peso papel }+ \text { muestra })-(\text { Peso papel solo })} \times 100 \\
\text { \% E.E. Base Seca. }=\frac{100 \times \% \text { E.E. }}{\% \text { M S }}
\end{gathered}
$$




\subsection{Determinación de cenizas}

Para la técnica determinación de cenizas se siguió el procedimiento y métodos de las técnicas antes descritas para laboratorio. Determinar la cantidad de residuo inorgánico que queda después de haber calcinado un residuo orgánico mediante el método de calcinación en mufla a $560^{\circ} \mathrm{C}$.

La muestra de un alimento se incinera a $550^{\circ} \mathrm{C}$. para quemar todo el material orgánico presente en la muestra. El material inorgánico que no se quema a ésta temperatura se denomina cenizas.

\subsubsection{Cálculo y expresión de resultados}

$$
\% \text { de cenizas }=\frac{(P C+C)-(P C \text { solo })}{(P C+M)-(P C \text { solo })} \times 100,
$$

donde: PC: Peso del crisol; C: Cenizas; M: Muestra.

$$
\% \text { de cenizas en base seca }=\frac{100 \times \% \text { de ceniza }}{\% \text { de materia seca }}
$$

\subsection{Determinación de materia orgánica}

$\%$ de Materia Orgánica $=100-\%$ de cenizas.

\subsection{Determinación fibra cruda}

Determinar y cuantificar la cantidad de fibra cruda presente en una muestra de alimento para consumo animal, bajo la metodología del análisis proximal para evaluar la calidad del mismo.

Este método se basa en la digestión ácida y alcalina de la muestra hidrolizando las proteínas, grasas y la mayoría de los carbohidratos obteniéndose un residuo de fibra cruda y sales que con calcinación posterior se determina la fibra cruda, simulando así el proceso de digestión que ocurre normalmente dentro del aparato digestivo de los animales.

\subsubsection{Cálculo y expresión de resultados}

$$
\begin{gathered}
\text { \% F.C. }=\frac{W \text { crisol con muestra digerida }-W \text { del crisol con cenizas }}{W \text { papel con muestra }-W \text { del papel solo }} \times 100 \\
\text { \% F.C. Base Seca. }=\frac{100 \times \% \text { Fc. }}{\% \text { de la M.S. }}
\end{gathered}
$$




\subsection{Determinación de humedad higroscópica}

Determinar y cuantificar la humedad higroscópica presente en una muestra de alimento completo o de un forraje, mediante la metodología del análisis proximal para evaluar y asegurar la calidad del forraje.

Este método se basa en la volatilización del agua a causa del calor, hasta que se haya eliminado el 100\% de agua aplicando para ello una temperatura de 105 grados centígrados.

\subsubsection{Cálculo y expresión de resultados}

$$
\begin{aligned}
\% \text { Materia seca }= & \frac{(\text { Peso del Crisol }+ \text { Muestra Seca })-(\text { Peso del Crisol })}{(\text { Peso del Crisol }+ \text { Muestra fresca })-(\text { Peso Crisol })} \times 100 \\
& \% \text { Humedad }=100-\% \text { Materia seca } .
\end{aligned}
$$

\subsection{Determinación de proteína bruta}

Determinar y cuantificar la cantidad de proteína bruta presente en una muestra de alimento seco o de un forraje, mediante la metodología del análisis proximal para evaluar la calidad del forraje o el alimento completo.

Calentando el alimento con ácido sulfúrico concentrado, los hidratos de carbono y las grasas se destruyen hasta formar anhídrido carbónico y agua. La proteína se descompone con la formación de amoníaco el cual interviene en la reacción con el ácido sulfúrico y forma sulfato de amonio.

$$
\mathrm{NH} 2 \mathrm{CH} 2 \mathrm{COOH}+3 \mathrm{H}_{2} \mathrm{SO} 4 \ldots . . \mathrm{NH} 3+2 \mathrm{CO} 2+3 \mathrm{SO}_{2}+4 \mathrm{H} 2 \mathrm{O}
$$

$2 \mathrm{NH} 3+\mathrm{H} 2 \mathrm{SO} 4$ Sulfato de amonio.

El sulfato de amonio en medio ácido es resistente y su destrucción con desprendimiento de amoníaco sucede solamente en medio básico. Por consiguiente, luego de la forma de la sal de sulfato de amonio, actúa en base fuerte al 50\% y se desprende todo el nitrógeno en forma de amoníaco:

$$
\begin{aligned}
& (\mathrm{NH} 4) 2 \mathrm{SO} 4+2 \mathrm{NaOH} \ldots \ldots \ldots . . . \mathrm{Na} 2 \mathrm{SO} 4+2 \mathrm{NH} 4 \mathrm{OH} \\
& 2 \mathrm{NH} 4 \mathrm{OH} \text {.............................. } 2 \mathrm{NH} 3+2 \mathrm{H} 2 \mathrm{O}
\end{aligned}
$$

El amoníaco que se desprende se calcula mediante la absorción de este con 0.1N de una solución de ácido clorhídrico por titulación.

\subsubsection{Cálculo y expresión de resultados}

$$
\begin{gathered}
\text { \% P.B. }=\frac{\mathrm{HCl} 0.1 \mathrm{~N} \text { estandarizado } \times 0.014 \times 6.25 \times \mathrm{ml} . \mathrm{HCl} 0.1 \text { Gastados. }}{\text { (peso papel }+ \text { muestra })-(\text { peso papel) }} \\
\text { \% P.B. en base seca }=\frac{100 \times \% \text { P.B. }}{\% \mathrm{MS} .}
\end{gathered}
$$




\section{Resultados y Discusiones}

Los valores promedios de la composición química de la alfalfa (Medicago sativa) a los 60 días de corte se observan en la Tabla 1. La humedad, materia seca (MS) y materia orgánica (MO) estuvieron dentro de los rangos de 9,78 $\pm 1,11 \% ; 90,22 \pm 1,11 \%$ y 89,47 $\pm 0,93 \%$, en base a MS, respectivamente; además, las cenizas, proteína bruta, fibra bruta, extracto etéreo, extractos libres de nitrógeno, fibra neutro detergente, fibra ácido detergente y lignina ácido detergente fueron de 10,53 \pm 0,93\%; 20,25 \pm 1,49\%; 35,22 \pm 3,96\%, $1,94 \pm 0,93 \% ; 32,06 \pm 2,11 \% ; 46,96 \pm 3,78 \% ; 40,67 \pm 3,36 \%$ y $8,23 \pm 0,64 \%$ en base a materia seca, respectivamente.

Al analizar la Tabla 2, del análisis bromatológico a los 60 días de corte en las diferentes zonas de la región Sierra - Centro, se puede observar que el mayo promedio de la humedad total fue $11,12 \pm 0,20 \%$ en (Rp-00542) mientras que el menos fue 7,79 \pm 0,05\% en (Rp-00224); para Materia Seca-Total los valores se obtuvieron entre 91,25 \pm 0,03\% (Rp336) y 88,53 \pm 0,09\% (Rp492); Cenizas base seca 12,51 $\pm 0,08 \%$ (Rp500) y 9,16 \pm 0,08\% (Rp521); el mayor valor para \% materia orgánica base seca fue de 90,84 \pm 0,13\% (Rp521) y el menor con 87,49 \pm 0,08\% (Rp521); para \% proteína cruda base seca la mejor respuesta fue $22,44 \pm 0,26 \%(R p 492) \%$ y el menor $18,73 \pm 0,08 \%(R p 336)$ fibra cruda base seca; $1,94 \pm 0,93 \%$ extracto etéreo base seca; 32,06 $\pm 2,11 \%$ extracto libre de nitrógeno base seca; 46,96 $\pm 3,78 \%$ fibra detergente neutro base seca; 40,67 $\pm 3,36 \%$ fibra detergente ácida base seca; $8,23 \pm 0,64 \%$ lignina detergente ácida base seca.

Los resultados analíticos de la composición química correspondientes al cultivo de alfalfa expuestos en la Tabla 1, muestran que los valores medios de la proteína bruta $(\mathrm{PB})$, extracto etéreo (EE), cenizas, fibra ácido detergente (FAD), lignina ácido detergente (LAD) y materia seca (MS) son similares a los expuestos por [13], quienes muestran contenidos de 90,7; 18,8; 34,6; 7,8; 9,1 y 2,28\% de MS, PB, FAD, LAD, Cenizas y EE en base a MS, respectivamente. Sin embargo, se observa que la alfalfa en este estudio contenía una menor concentración de EE (1,94\% en MS) y más PB $(20,25 \%$ en MS), FAD $(40,67 \%$, en MS) y LAD (8,23\%, en MS) en comparación a las observadas por [14] quienes obtuvieron valores medios de $26 \%$ de PB, $30 \%$ de FND y $14 \%$ de FAD de cinco muestras de alfalfa. Las diferencias en los resultados de [14] con el presente estudio pueden estar asociados con el tiempo de cosecha y la edad de la planta [15]

Por sus características nutricionales la alfalfa es el principal forraje de sustento en la producción ganadera del país y del mundo [16], por lo tanto, el constante uso de este forraje viene siendo de mayor rentabilidad en la ganadería ecuatoriana. La disposición como pasto fresco, a su vez como henos de alta calidad o con mezclas forrajeras, ha demostrado un total desempeño en los rendimientos nutricionales [17]. Los henos de alfalfa recolectados de la zona Sierra-Centro del país (Tabla 2), demuestran lo expuesto.

Las diferencias entre los valores medios de PB fueron 18,73 a $22,44 \%$ en MS, lo cual supone una diferencia del contenido de PB entre los diferentes henos de mezclas de forrajes con alfalfa, aproximadamente en un 4\%. La concentración EE en todos los henos fueron similares, sin embargo, se muestran claras diferencias. Las muestras Rp-00492 mostraron concentraciones más altas en comparación con las muestras que contenían 


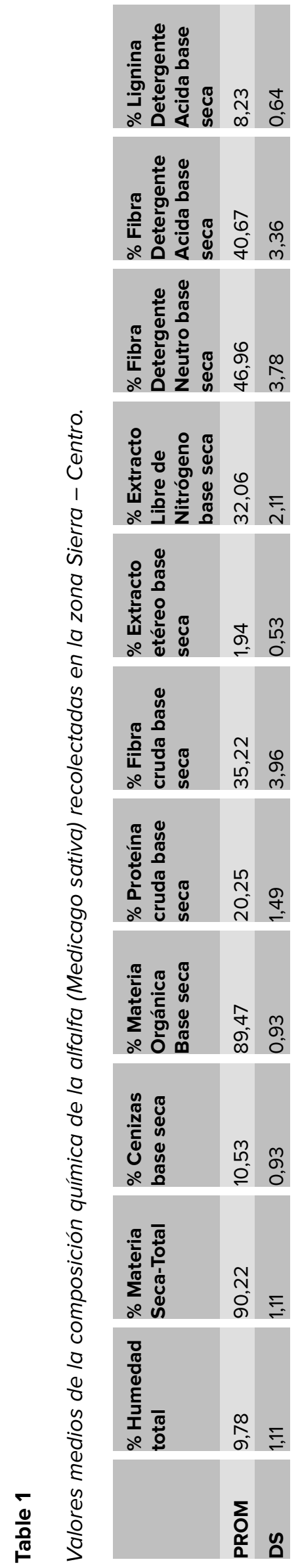




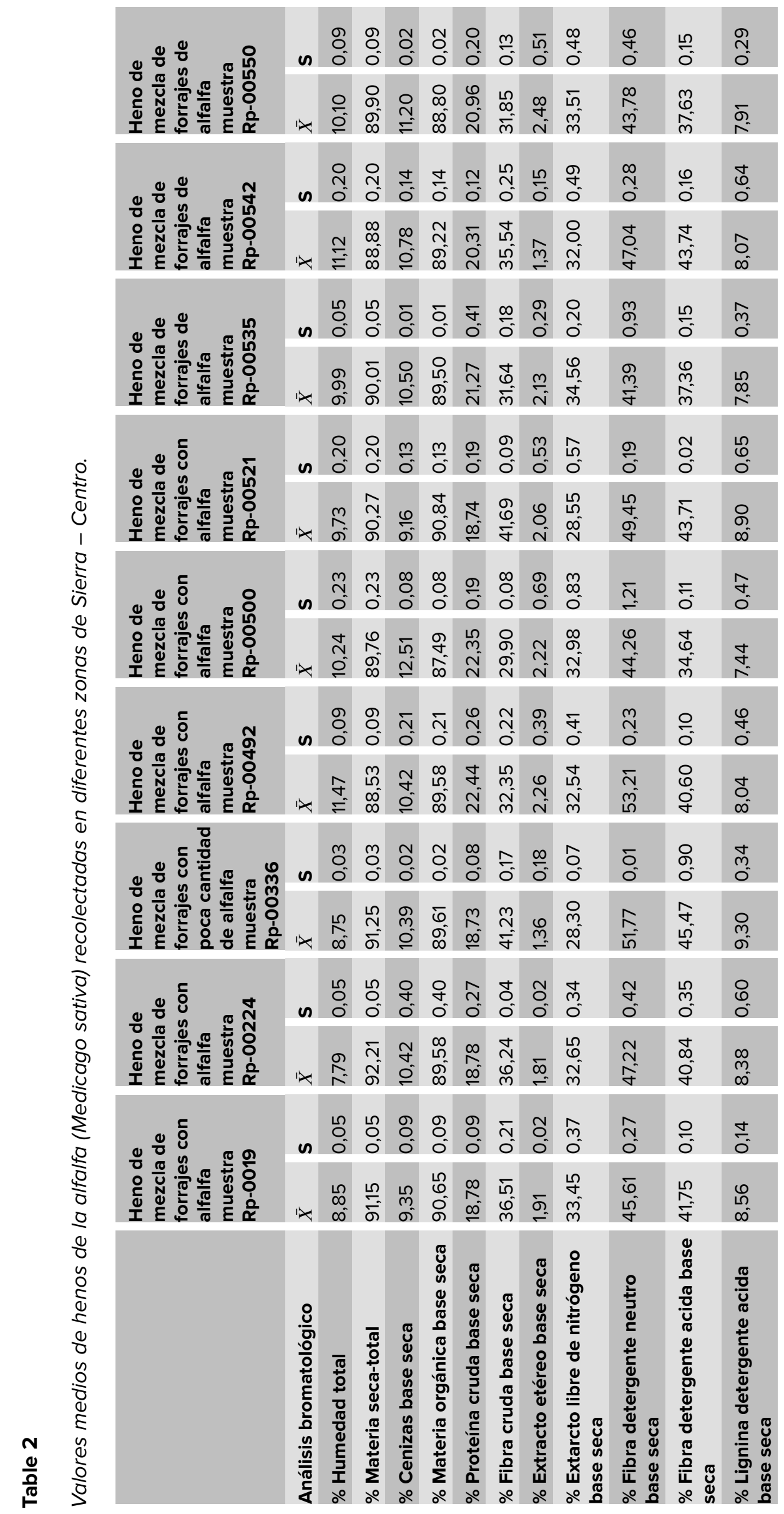


poca cantidad de alfalfa (Rp-00336) mostrando menos concentraciones de EE (2,26 y $1,36 \%$ de EE en MS, respectivamente) probablemente a que el tipo de cultivo ejerce poca influencia sobre el contenido en EE de la alfalfa, por lo que, existen otros factores como el estado de madurez y desarrollo de la planta, cortes y tiempo de cultivo [18].

Los valores medios en los contenidos de FND de los henos de mezclas de forrajes con alfalfa estuvieron entre 41,39 y 53,21\% de FND en MS para las muestras Rp-00492 y Rp-00535 respectivamente, supone una diferencia del contenido aproximadamente de $11,82 \%$. Para los henos de mezclas forrajeras con alfalfa su contenido en FAD y LAD va aumentando según se reduce la cantidad de alfalfa en la producción del heno entre $45,47 \%$ de FND y $9,30 \%$ en MS de LAD en MS y $34,64 \%$ de FAD y $7,44 \%$ en MS de LAD en MS.

Los valores medios, fueron similares a los obtenidos por [18] quienes muestran concentraciones similares de PB, EE, FAD y LAD, cenizas y MS para los henos de alfalfa en estado de desarrollo; oscilando los valores medios entre un 83,91 y $86,73 \%$ de MS, 10,36 y $13,60 \%$ de ceniza, 1,08 y $2,51 \%$ de $E E, 19,66$ y $35,06 \%$ de FAD, 3,47 y $6,49 \%$ de LAD y 16,97 y $28,23 \%$ de PB en MS.

El comportamiento de los henos con relación a su contenido en cenizas difirió entre las muestras Rp-00521 con menor contenido en cenizas de 9,16\% en MS a diferencia de la muestra Rp-00500 con un contenido mayor sobre las muestras de heno de un $12,51 \%$ de ceniza en MS. La proporción de cenizas fue bastante similar en las nueve muestras de heno de mezcla de forraje con alfalfa. Además, conviene recordar que las muestras recogidas pudieran haber estado contaminadas en mayor o menor grado por partículas procedentes del terreno donde se realizó el cultivo, hecho que puede extremar los resultados obtenidos, por ello, el valor real de los datos sobre el contenido en cenizas puede verse reducido.

En este ensayo, en definitiva, la composición química de los henos de mezclas de forrajes con alfalfa, parecen estar en acuerdo con diferentes estudios $[15,18,19]$ en sentido de que pueden estar influenciadas por la precocidad de los cultivaros, tiempo de corte y el porcentaje de mezcla, además, de la zona de siembra y tiempo de cosecha [20]. Sin embargo, una falta de concordancia entre nuestros resultados y los obtenidos por otros estudios es más aparente que real.

\section{Conclusiones}

Por lo que se puede determinar que la mezcla forrajera aporta todos los nutrientes a todas las especies de interés zootécnico, ya que presentaron mejor rendimiento productivo y calidad nutricional, principalmente en el contenido de PC, ENL y DIG, las cuales son las características nutritivas de consideración en la formulación de alimentos para rumiantes.

\section{References}

[1] Vásquez HA et al. El uso eficiente de los forrajes tropicales en la alimentación de los bovinos. Revista Ciencia Animal. 2014;7:111-132. 
[2] Trujillo G. Guía para la utilización de recursos forrajeros tropicales para la alimentación de bovinos. Comité de Ganaderos del Huila. 2009:17.

[3] Schwab EC et al., Estimating silage energy value and milk yield to rank corn hybrids. Animal Feed Science and Technology. 2003;109(1-4):1-18.

[4] Baizán S et al. Alternativas forrajeras sostenibles como cultivo invernal en zonas templadas. Pastos: Revista de la Sociedad Española para el Estudio de los Pastos. 2015;45(2):23-32.

[5] Sahay G et al. Conservation Agriculture. Springer; 2016. Role of genetic resources of forages in the present changing climatic scenario. p. 159-183.

[6] Santamaría Cesar J et al., Evaluación del modelo Epic para estimar el potencial productivo de alfalfa (medicago sativa L.) en diferentes ambientes ecológicos de México. Técnica pecuaria en México. 2000;38(2):151-161.

[7] Bouton J. The economic benefits of forage improvement in the United States. Euphytica. 2007;154(3):263-270.

[8] Cueto WJ, Quiroga GH, Becerra MC. Nitrógeno disponible y desarrollo del ballico anual. 1. Producción, calidad del forraje y acumulación de nitratos. Terra. 2003;21:285-295.

[9] Laurin $\mathrm{M}$ et al. El papel de la agricultura ecológica en la disminución del uso de fertilizantes y productos fitosanitarios químicos. 2006. Agroecologia. Recuperado de: www.agroecologia.net/ recursos/publicaciones/publicacionesonline/2006/CD\{\%\}20Congreso\{\%\}20Zaragoza/Ponencias/ 105\{\%\}20Laurin\{\%\}20Com-\{\%\}20El\{\%\}20papel.pdf, 2006.

[10] Castro Bedriñana J, Peinado DC, Schwartz PL. Evaluación del compost de guano de pollo en el rendimiento y calidad nutricional de la alfalfa en la sierra central del Perú. Revista de Investigaciones Veterinarias del Perú. 2019;30(4):1562-1568.

[11] Williams S. Official methods of analysis. Association of Official Analytical Chemists; 1984

[12] FAO. Food and N. Agriculture Organization of the United, FAO food and nutrition paper. Food and Agriculture Organization of the United Nations; 1979.

[13] Avendaño J, et al. Ovinos alimentados con raciones que incluyen tagasaste (chamaecytisus proliferus subsp. palmensis) en reemplazo de heno de alfalfa.: II. Digestibilidad y Consumo de Nutrientes. Agricultura Técnica. 2004;64(3):271-279.

[14] Stefanon B, Pell AN, Schofield P. Effect of maturity on digestion kinetics of water-soluble and waterinsoluble fractions of alfalfa and brome hay. Journal of Animal Science. 1996;74(5):1104-1115.

[15] Van Soest PJ. Nutritional ecology of the ruminant. Cornell university press; 2018.

[16] Capacho-Mogollón AE, Flórez-Delgado DF, Hoyos-Patiño JF. Biomasa y calidad nutricional de cuatro variedades de alfalfa para introducir en Pamplona, Colombia. Ciencia y Agricultura. 2018;15(1):61-67.

[17] Vázquez-Vázquez C, et al. Alfalfa (Medicago sativa L.) forage nutritional value and yield at different cattle manure doses. Revista Mexicana de Ciencias Pecuarias. 2010;1(4):363-372.

[18] Alvir MR, González J, Argamentería A. Degradación ruminal de la proteína de diferentes henos de alfalfa. Pastos. 2011;15(1-2):193-200.

[19] Fernández CR, Dova SW, de Lucas Burneo JJ. Particularidades anatómicas, fisiológicas y etológicas con repercusión terapéutica, en medicina aviar (II): aparato digestivo, aparato cardiovascular, sistema músculo-esquelético, tegumento y otras características. Panorama actual del medicamento. 2017;41(401):223-234.

[20] Berg BP et al., Bloat in cattle grazing alfalfa cultivars selected for a low initial rate of digestion: A review. Canadian Journal of Plant Science. 2000;80(3):493-502. 